\title{
Redes Sociais e Complexas: um modelo computacional para a investigação da pós- graduação Brasileira em Ensino de Física
}

\author{
Nascimento, J. O. do. ${ }^{1 *}$; Pereira-Guizzo, C. S.; Moreira, D. M.; Monteiro, R. L. S.; \\ Pereira, H. B. B.; Moret, M. A. \\ 1 Programa de Modelagem Computacional, Faculdade de Tecnologia SENAI CIMATEC, Salvador, BA, Brasil. \\ 2 Universidade do Estado da Bahia, Salvador, Bahia, Brasil.
}

*e-mail: jeffersonascimento@gmail.com

\begin{abstract}
Resumo
Por meio da utilização dos índices pertencentes a teoria de redes sociais e complexas é possível a análise de propriedades emergentes em redes semânticas. Este artigo tem como objetivo descrever e analisar uma rede semântica formada pelas palavras-chave pertencentes aos trabalhos de mestrado, doutorado e livre docência realizados na área de Ensino de Física, entre os anos de 1996 à 2006, no Brasil. Para alicerçar o estudo realizamos os cálculos e analisamos os índices pertences às redes sociais e complexas. Também apresentamos a metodologia utilizada à construção da rede, em que as palavraschave receberam um tratamento prévio criterioso antes de um segundo passo, que é por meio da utilização de softwares. Percebemos indicações de que a rede apresentou a topologia livre de escala e 0 fenômeno pequeno mundo. Por fim, as análises indicaram também que a maioria das temáticas dos hubs emergentes na rede, estão relacionados para formação do Físico e não em metodologias para o Ensino de Físicas nos diversos níveis de ensino.
\end{abstract}

\begin{abstract}
With the indexes belonging to theory and complex social networks, it is possible to analyze emergent properties in semantic networks. This article aims to describe and analyze a semantic network formed by keywords, belonging to the master's works, doctoral and free teaching made in the area of Physical Education, between 1996-2006, in Brazil. In support of the study, we conducted calculations and analyze the belongings indices of social and complex networks. We also present the methodology used to construct the network, where the keywords given a careful pretreatment before a second step is with software. We noticed indications that the network presented the free topology of scale and the small world phenomenon. Finally, the analysis also indicated that most of the themes of the emerging hubs in the network are related to formation of the physics' teacher and not on methodologies for Physical Education in different educational levels.
\end{abstract}

Keywords (Palavras chaves): Complex Networks, Social Networks, Physics Education.

\section{Introdução}

A teoria de redes como possibilidade de estudo de entidades conectadas, ocorre por meio da teoria dos grafos e as nuances pertencentes às redes sociais e complexas. Com a utilização das métricas da teoria de redes é possível a caracterização e a investigação do comportamento das supracitadas entidades, observando as suas propriedades emergentes. Diante deste cenário o estudo de padrões e/ou modelos de previsões, por exemplo, em estruturas compostas por atores conectados têm apresentado uma frequência maior de realização. Nesse sentido, a utilização de ferramentas computacionais cada vez mais robustas e sofisticadas, têm sido ampliadas. Verificam-se, como exemplos dessas estruturas, indivíduos, organizações, páginas webs, computadores, palavras em títulos, neurônios, redes de citações, redes de coautoria, etc. [1].

Neste artigo, apresentamos uma rede semântica construída pelas palavras-chave de dissertações, teses de doutorado e livre docência em Ensino de Física no Brasil, entre os anos de 1996 e 2006. Indicamos a distribuição de graus realizada e as possíveis 
contribuições da aplicação da Teoria de Redes Sociais e Complexas aos padrões estruturais semânticos, verificados nas referidas palavras-chave, para o Ensino de Física nacional.

Este trabalho está organizado em 4 seções. $\mathrm{Na}$ segunda, apresentaremos os materiais e métodos utilizados para a construção da rede. Na terceira, os resultados e as discussões consequentes à rede semântica originada são discutidos. Na quarta seção, as considerações finais do presente artigo.

\section{Materiais e métodos}

Este artigo tem como finalidade realizar a construção de uma rede semântica das palavras-chave pertencentes as dissertações, teses de doutorado e livre docência especificamente na temática de Ensino de Física. O período de análise correspondeu de 1996 à 2006, conforme informações disponíveis em Salem e Kawamura [2]. Consequentemente, também verificar as propriedades emergentes na rede e, caracterizá-la conforme a sua topologia.

Para alcançarmos os objetivos, iniciamos com a abordagem de grafo $G=(V, E)$, que corresponde a uma estrutura matemática que consistente em dois conjuntos: $V$ (finito e não vazio) e $E$ (relações binárias sobre V) [3]. Os elementos de V são denominados de vértices e os elementos de $E$, são as arestas. $O$ primeiro conjunto (V) é finito e não vazio e o segundo (E), corresponde as possíveis interações binárias sobre $\mathrm{V}$.

As propriedades observadas das redes complexas, para análise da rede semântica de palavras-chave deste artigo, correspondem àquelas relacionadas aos índices de estatística básica. Com estas informações, é possível caracterizar a topologia da rede, como sendo, por exemplo, aleatória (Randon Network), mundo pequeno (Small-Word) ou livres de escala (Scale Free). Portanto, para o estudo da rede semântica proposta, observar-se-ão as seguintes propriedades: número de vértices $(n)$, número de arestas $(E)$, grau médio $(<k>)$, densidade $(\Delta)$, coeficiente de aglomeração $\left(\mathrm{C}_{\mathrm{v}}\right)$ e coeficiente de aglomeração médio $\left(\mathrm{C}_{\mathrm{ws}}\right)$ caminho mínimo médio (L) e diâmetro (D), especificados da seguinte maneira:

Número de vértice $(n)$ : Corresponde a cardinalidade do conjunto de vértices da rede

$$
n=|V|
$$

Número de vértices $(\mathrm{m})$ : Corresponde a cardinalidade do conjunto de arestas da rede

$$
m=|E|
$$

Grau médio ( $\langle\mathrm{k}\rangle)$ : Representa a quantidade média de conexões em cada vértice em uma rede e, é obtido da seguinte maneira:

$$
\langle k\rangle=\frac{1}{n} \sum_{i}^{n} k_{i}
$$

Densidade $(\Delta)$ : É o total de arestas dividas pelo número máximo possível de arestas:

$$
\Delta=\frac{m}{n(n-1) / 2}
$$

Esta propriedade está relacionada com a coesão na rede.

Coeficiente de aglomeração Médio $\left(\mathrm{C}_{\mathrm{ws}}\right)$ : O coeficiente de aglomeração médio dos vértices pertencentes a uma rede:

$$
C_{w s}=\frac{1}{N} \sum_{V=1}^{N} C_{V}
$$

O coeficiente de aglomeração de um vértice $V$, denominado de $\mathrm{C}_{\mathrm{V}}$, mede a proporção de arestas entre os vizinhos do vértice $\mathrm{V}, \mathrm{Ev}$, e o máximo número de arestas possíveis,

$$
C_{V}=\frac{2 E_{V}}{k_{V}\left(k_{V}-1\right)}
$$

Este índice apresenta a extensão em que os amigos de um indivíduo são amigos uns com os outros.

Caminho mínimo médio ou distância geodésica (L):

$$
L=\frac{1}{n(n-1)} \sum_{i \neq j} d_{i j}
$$

O termo $\mathrm{d}_{i j}$ corresponde a distância geodésica, em termos do número de arestas existentes entre os vértices $i$ e $j$;

Diâmetro (D): É a maior distância geodésica da rede, calculada da seguinte maneira:

$$
D=\max _{(i, j)} d_{i j}
$$

Em relação às medidas de redes sociais, as análises realizadas foram por meio das observações das centralidades de grau. Esse índice indica a importância que um determinado vértice tem, por meio de suas conexões, com vértices vizinhos conectados a ele [1]. Para que a rede pudesse ser construída, realizamos um tratamento manual nas palavras-chave. Em um arquivo de texto, cada linha recebeu um único conjunto das palavras-chave pertencentes a cada trabalho Stricto Sensu (correspondendo a uma sentença). Na sequência, utilizamos as regras de formatação, descritas no Quadro 1.

Quadro 1: Regras para a formatação das palavras-chave. Fonte: Adaptado de Pereira et al. [3].

\begin{tabular}{|l|l|}
\hline 1 & $\begin{array}{l}\text { Cada conjunto de palavras-chave corresponde a } \\
\text { uma sentença; }\end{array}$ \\
\hline 2 & $\begin{array}{l}\text { Os sinais gráficos de cada sentença como o ponto } \\
\text { e vírgula, ponto de interrogação, ponto de } \\
\text { exclamação e reticências são eliminados; }\end{array}$ \\
\hline 3 & $\begin{array}{l}\text { Os nomes devem formar uma palavra simples. } \\
\text { "Mecânica Estatística", respectivamente, tornar- }\end{array}$ \\
\hline
\end{tabular}




\begin{tabular}{|l|l|}
\hline & $\begin{array}{l}\text { se-ão: "aprendizagemsignificativa" } \\
\text { "mecânicaestatística". }\end{array}$ \\
\hline 4 & $\begin{array}{l}\text { Números ordinais devem ser escritos da seguinte } \\
\text { forma, por exemplo, "8a" e "10", tornar-se-ão, } \\
\text { respectivamente: "oitava" e "primeiro". }\end{array}$ \\
\hline 5 & $\begin{array}{l}\text { Os números devem ser escritos de forma textual, } \\
\text { por exemplo, "1972" e "1995", tornar-se-ão } \\
\text { "umnovesetedois" e "umnovenovecinco". }\end{array}$ \\
\hline 6 & $\begin{array}{l}\text { Palavras compostas devem ser consideradas } \\
\text { como uma só palavra, por exemplo, "ensino de } \\
\text { ciências", tornar-se-á, "ensinodeciências". }\end{array}$ \\
\hline 7 & $\begin{array}{l}\text { Palavras escritas de formas incorretas, devem ser } \\
\text { corrigidas. }\end{array}$ \\
\hline 8 & $\begin{array}{l}\text { As palavras que se repetem no conjunto de } \\
\text { palavras-chave, devem ser retiradas, } \\
\text { permanecendo apenas uma. }\end{array}$ \\
\hline 9 & $\begin{array}{l}\text { Linguagem especializada deve ser mantida, } \\
\text { sempre que possível. }\end{array}$ \\
\hline 10 & $\begin{array}{l}\text { Palavras que apresentam mútua importância, } \\
\text { devem se tornar apenas uma, por exemplo, } \\
\text { "Física Quântica", tornar-se-á, "FísicaQuântica". }\end{array}$ \\
\hline 11 & $\begin{array}{l}\text { Palavras escritas em linguagem diferente do } \\
\text { Português, devem ser traduzidas. }\end{array}$ \\
\hline
\end{tabular}

No que se refere ao tratamento por meio de recursos computacionais, foram utilizados os procedimentos em conformidade com [1,4-5]. Cabe ressaltar que as ambiguidades eliminadas, seguindo 0 algoritmo desenvolvido em [5].

\section{Resultados e Discussão}

Por meio da metodologia referenciada no tópico anterior, foi possível a construção da rede semântica apresentada na Figura 1:

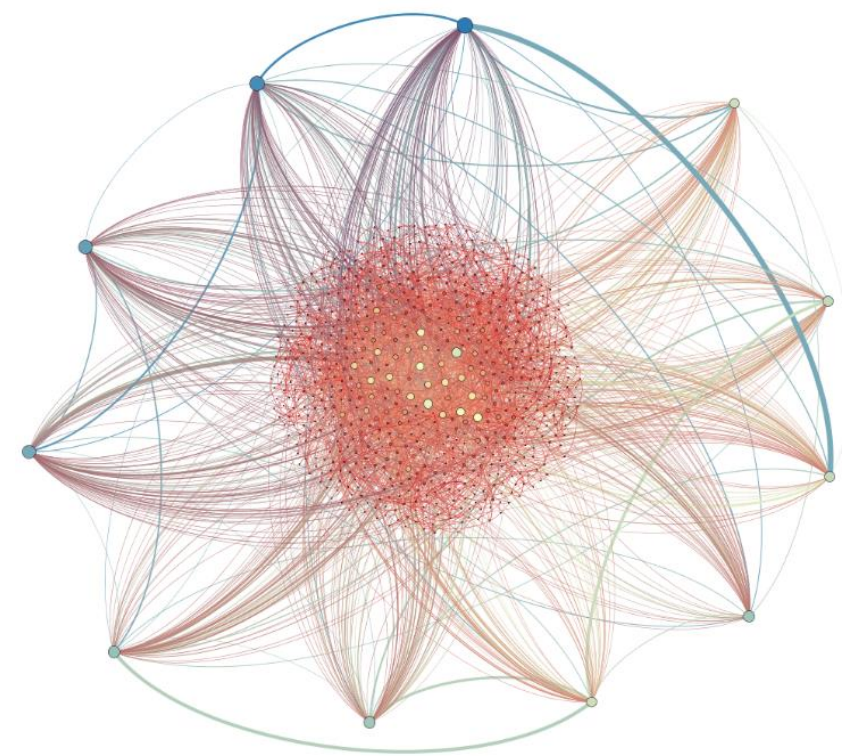

Figura 1: Rede semântica das palavras-chave dos trabalhos em Ensino de Física. Fonte: Dos autores, 2016.
$\mathrm{Na}$ Tabela 1, apresentamos as propriedades obtidas em relação a rede semântica das palavras-chave (Figura 1), relativo aos índices de redes complexas:

Tabela 1: Propriedades pertences à Rede Semântica

\begin{tabular}{|c|c|c|c|}
\hline Propriedade & Valor & Propriedade & Valor \\
\hline $\boldsymbol{n}=|\boldsymbol{V}|$ & 669 & Maior Componente (\%) & 98,95 \\
\hline $\boldsymbol{m}=|\boldsymbol{E}|$ & 5910 & $\langle\boldsymbol{k}\rangle$ & 8,8 \\
\hline $\boldsymbol{\Delta}$ & 0,013 & $\boldsymbol{C}_{w s}$ & 0,74 \\
\hline $\boldsymbol{L}$ & 3,06 & $\boldsymbol{D}$ & 6 \\
\hline
\end{tabular}

Conforme a Tabela 1, observamos que o valor do caminho mínimo médio $(\mathrm{L})$ é de aproximadamente três arestas para conectarem duas palavras na rede. $O$ diâmetro (D), apresentou valor de seis, na rede. Tais informações refletem $o$ fato de que as palavras pertencentes as palavras-chave de trabalhos distintos, estão conectados entre si, no máximo, por meio de seis outras palavras.

A densidade $(\Delta)$ é uma medida que está relacionada com o nível de conectividade dentro da rede, ou seja, um indicativo do nível de coesão dos vértices [4]. Conforme Pereira et al. [4] esta informação em uma dada rede semântica de títulos, indica a quantidade de ligações entre as palavras, ou seja, uma "tendência dos títulos estarem ligados através de um número grande de palavras", pensamento este que pode ser aplicado também para a rede semântica de palavras-chave (Figura 1). O valor verificado é da ordem de 1,3\%. Assim é possível que na rede semântica apresentada, haja um grande número de palavras conectando as cliques, tornando a rede espaça, com baixa densidade. Desta forma, a densidade encontrada pode corresponder a uma menor coesão entre as conexões na rede.

O valor verificado para o coeficiente de aglomeração $\left(\mathrm{C}_{\mathrm{ws}}\right)$, foi da ordem de $75 \%$, indicando uma alta conectividade entre as palavras presentes da rede semântica.

Em relação a caracterização da topologia da rede semântica, a distribuição de graus realizada, é a exposta na Figura 2. Ao se realizar um ajuste linear, o padrão verificado segue uma lei de potência, da forma $\mathrm{P}(\mathrm{k}) \sim \mathrm{k}^{-\mathrm{v}}$. 


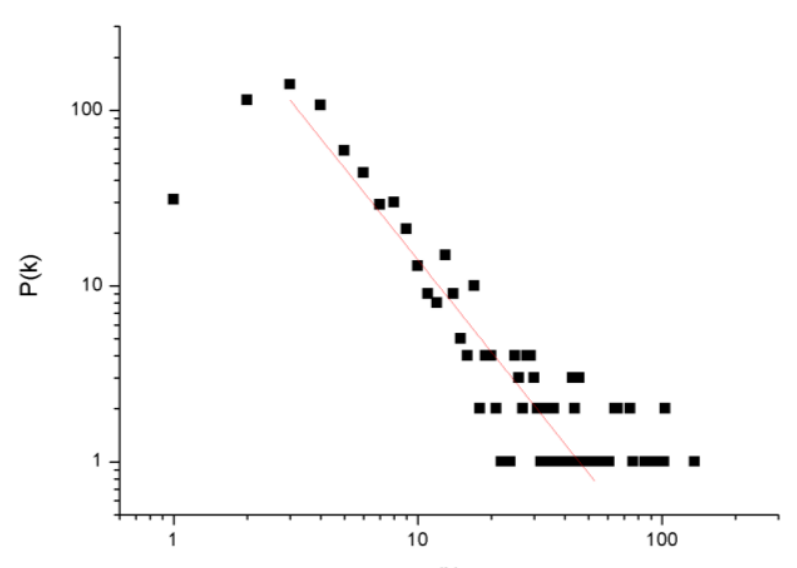

(k)

Figura 2 - Distribuição de graus da rede semântica (com y = 1,73784 e o ajuste $R^{2}=0,85309$ )

De acordo com o gráfico da Figura 2, a distribuição de graus realizada, fornece indícios de uma rede livre de escala. Conforme Barabási [6], percebe-se então que possivelmente há, para a década investigada, uma adesão preferencial por certos vértices na rede (i.e. palavras-chave), indicando a presença de hubs. Assim, os vértices altamente conectados têm uma probabilidade maior em adquirir novas ligações do que aqueles que se apresentam menos conectados.

$\mathrm{Na}$ Tabela 2, realizamos uma comparação entre as redes semânticas de palavras-chave e a rede aleatória equivalentes dela (rede com o mesmo número de vértice e grau médio):

Tabela 2 - Propriedades: rede semântica de palavras-chave e a rede aleatória equivalente (random network)

\begin{tabular}{|c|c|c|c|c|}
\hline Rede & Propriedade & Valor & Propriedade & Valor \\
\hline \multirow{4}{*}{ Keywords } & $n=|V|$ & 669 & $\begin{array}{c}\text { Maior } \\
\text { Componente } \\
(\%)\end{array}$ & 98,95 \\
\hline & $m=|E|$ & 5910 & $\langle\boldsymbol{k}\rangle$ & 8,8 \\
\hline & $\Delta$ & 0,013 & $C_{w s}$ & 0,74 \\
\hline & $L$ & 3,06 & $D$ & 6 \\
\hline \multirow{4}{*}{$\begin{array}{l}\text { Random } \\
\text { Network }\end{array}$} & $n=|V|$ & 669 & $\begin{array}{c}\text { Maior } \\
\text { Componente } \\
(\%)\end{array}$ & 98,95 \\
\hline & $\boldsymbol{m}=|E|$ & 3002 & $\langle\boldsymbol{k}\rangle$ & 8,8 \\
\hline & $\Delta$ & 0,013 & $C_{w s}$ & 0,012 \\
\hline & $L$ & 3,02 & $D$ & 6 \\
\hline
\end{tabular}

Percebemos que as redes apresentaram o mesmo valor aproximado para o caminho mínimo médio (L). Observamos também que o diâmetro da rede semântica e a rede equivalente, são os mesmos. Os coeficientes de aglomeração $\left(\mathrm{C}_{\mathrm{ws}}\right)$ da rede semântica de palavras-chave, apresentou valor elevado em comparação com a rede aleatórias equivalentes. Então, diante destes resultados verificados na comparação, que estão com de acordo com o método proposto por Watts e Strogatz [7], percebemos que a rede semântica (Figura 1), apresenta o fenômeno mundo pequeno (Small-World).

De acordo com Pereira et al. [4], Caldeira et al. [5] e Santos Junior et al. [8], os dados da Tabela 2, para a rede das palavras-chave, também são indicadores para o padrão verificado na Figura 2: uma rede semântica caracterizada como livre de escala Em relação ao coeficiente $\gamma$ verificado na Figura 2, é menor do que normalmente é indicado na literatura científica e o proposto por Barabási e Albert [9]: $2,1 \leq \gamma \leq 4$. Contudo, as análises de redes semânticas realizadas por Fadigas et al. [1] apresentaram $\gamma \leq 2,2$ e $\gamma<2$, na pesquisa de Seyed-allaei et al. [10]. Há uma preferência na rede por determinados vértices (hubs), conforme Quadro 2:

Quadro 2: Centralidades de grau dos 10 primeiros hubs da rede.

\begin{tabular}{|c|c|}
\hline Vértices (V) & Grau (k) \\
\hline História da Ciência & 102 \\
\hline Livro didático & 97 \\
\hline Atividade experimental & 89 \\
\hline Linguagem & 85 \\
\hline Formação continuada de professores & 76 \\
\hline Formação de professores & 74 \\
\hline Proposta didática & 74 \\
\hline Filosofia da ciência & 67 \\
\hline Concepção do professor & 66 \\
\hline Mecânica & 64 \\
\hline
\end{tabular}

O Quadro 2 traz como exemplo, os dez primeiros vértices com maiores medidas de graus na rede. Este fato nos permite inferir sobre a importância (centralidade de grau) que um determinado nó tem, por meio de suas conexões, com os nós imediatamente conectados a ele (vértices vizinhos) [1]. As informações retratam, que para a rede de palavras-chave, o vértice com maior centralidade de grau foi "história da ciência". A palavras "livro didático", "atividade experimental" e "linguagem", seguem como segundo, terceiro e quarto vértices, respectivamente, em termos de importâncias na rede. Seguindo este raciocínio, no Quadro 3, estão relacionados 10 vértices, selecionados pelos seus respectivos números de graus, que podem representar tópicos importantes relacionados aos conteúdos de física.

Quadro 3: Centralidades de grau de vértices que representam temáticas importantes em Física.

\begin{tabular}{|c|c|}
\hline Vértices (V) & Grau (k) \\
\hline Mecânica & 64 \\
\hline
\end{tabular}




\begin{tabular}{|c|c|}
\hline Física moderna e contemporânea & 53 \\
\hline Astronomia & 47 \\
\hline Eletromagnetismo & 44 \\
\hline Óptica & 33 \\
\hline Gravitação & 26 \\
\hline Termodinâmica & 25 \\
\hline Mecânica Quântica & 14 \\
\hline Relatividade Restrita & 12 \\
\hline Cosmologia & 9 \\
\hline
\end{tabular}

Os vértices contidos no Quadro 3 indicam que, tópicos considerados importantes para o ensino da disciplina física, foram pouco escolhidos como palavras-chave. $O$ vértice "Mecânica" ( $k=64)$ é o primeiro assunto que surge na rede semântica e apresenta grau de conexão $k=64$, ou seja, apresentam baixa centralidade de grau se comparados com os nove primeiros vértices contidos no Quadro 2. Estas informações podem indicar que novos trabalhos poderão ter, baixa probabilidade de ocorrerem com tópicos importantes no Ensino de Física.

\section{Considerações finais}

Por meio da análise da rede semântica formada pelas palavras-chave pertencentes aos trabalhos em Ensino de Física no Brasil (1996-2006), foram possíveis os cálculos dos índices da teoria de redes complexas e sociais. Também caracterizamos a topologia da rede e observamos vértices importante, que se destacaram nela. As propriedades obtidas indicaram que a rede semântica apresentou o fenômeno mundo pequeno (small-world) e também se caracterizou como uma rede livre de escala (scale free). Ambas as características são possíveis, pois, não são excludentes entre si.

A observação das palavras-chave com maiores centralidades de grau nos faz sugerir que, devido as características como o crescimento e a adesão preferencial da rede semântica construída e estudada, há uma maior probabilidade de ocorrem novas palavras-chave com as palavras do Quadro 2 do que as demais palavras do quadro 3 (com exceção ao vértice Mecânica).

A alta conectividade evidenciada nas redes, pelo coeficiente de aglomeração, os caminhos mínimos médios com os mesmos valores das redes aleatórias equivalentes e, uma distribuição de graus indicando uma lei de potência, norteiam também essa caracterização. Pelo fato delas serem resistentes à retirada de vértices de forma aleatória, apresentam vulnerabilidade em caso de retirada das palavras com maior medida de centralidade (Quadro 1). Assim, caso retirássemos da rede semântica (Figura 1) os vértices pertencentes ao Quadro 2, possivelmente apresentarse-ia dívida em componentes o que poderia dificultar a propagação de uma determinada informação. De acordo com a Ref. [1] esta dificuldade pode ocorrer em termos de não favorecer aos leitores, mecanismos de busca dos trabalhos, por palavras-chave. Ao serem observados os vértices com maiores centralidades, foi possível inferir que, a rede semântica de palavraschave, objetivaram conteúdos voltados para a formação de professores. Sugere-se também que a minorias dos trabalhos exploraram metodologias de Ensino de Física voltadas para os discentes.

\section{Agradecimentos}

JON agradece à FAPESB, referente ao apoio financeiro parcial recebido por meio da bolsa de doutorado (BOL170/2015). DMM e MAM agradecem ao CNPq pelo suporte financeiro parcial oriundo de suas bolsas de Produtividade em Pesquisa (No. 301591/2009-1) e (No. 304454/2014-1).

\section{Referências}

[1] I. S. Fadigas, T. H. P. Casas, V. Senna, M. A. Moret \& H. B. B. Pereira, Educação Matemática Pesquisa, 11, 167 (2009).

[2] S. Salem \& M. R. D. Kawamura, Ensino de Física no Brasil: catálogo analítico de dissertações e teses (1996-2006). São Paulo: Instituto de Física da USP / PROFIS, 2009.

[3] J. L. Gross \& j. Yellen, Graph theory and its applications. CRC press, Boca Raton FL USA 2005.

[4] H. B. B. Pereira, I. S. Fadigas, R. L. S. Monteiro, A. J. A. Cordeiro \& M. A. Moret, Physica A 390, 1192 (2011).

[5] S. M. G. CALDEIRA, T. Petit Lobão, R. F. S. Andrade, A. Neme \& J. G. V. Miranda, Eur. Phys. J. B 49, 523 (2006).

[6] A. L. Barabási Science 325, 412 (2009).

[7] D. J. Watts \& S. H. Strogatz, Nature 393, 440 (1998).

[8] R. P. Santos Júnior, A. A. A. O. Rodrigues, C. R. Lopes, I. P. Déjardin, J. L. B. Peixoto \& M. V. Cunha, DataGramaZero 15(3), 3 (2014).

[9] A. L. Barabási \& R. Albert, Science, 286, 509 (1999).

[10] H. Seyed-allaei, G. Bianconi \& M. Marsili, Phys. Rev. E 73, 046113 (2006). 